\title{
An Approach to Existence of Fixed Points of Generalized Contractive Multivalued Mappings of Integral Type via Admissible Mapping
}

\author{
Muhammad Usman Ali, ${ }^{1}$ Tayyab Kamran, ${ }^{2}$ and Erdal Karapınar ${ }^{3,4}$ \\ ${ }^{1}$ Department of Mathematics, School of Natural Sciences, National University of Sciences and Technology, H-12, \\ Islamabad 44000, Pakistan \\ ${ }^{2}$ Department of Mathematics, Quaid-i-Azam University, Islamabad 45320, Pakistan \\ ${ }^{3}$ Department of Mathematics, Atilim University, Incek, 06836 Ankara, Turkey \\ ${ }^{4}$ Nonlinear Analysis and Applied Mathematics Research Group (NAAM), King Abdulaziz University, Jeddah 21491, Saudi Arabia
}

Correspondence should be addressed to Muhammad Usman Ali; muh_usman_ali@yahoo.com

Received 3 June 2014; Revised 4 July 2014; Accepted 4 July 2014; Published 21 July 2014

Academic Editor: Poom Kumam

Copyright (C) 2014 Muhammad Usman Ali et al. This is an open access article distributed under the Creative Commons Attribution License, which permits unrestricted use, distribution, and reproduction in any medium, provided the original work is properly cited.

We investigate the existence of a fixed point of certain contractive multivalued mappings of integral type by using the admissible mapping. Our results generalize the several results on the topic in the literature involving Branciari, and Feng and Liu. We also construct some examples to illustrate our results.

\section{Preliminaries and Introduction}

Fixed point theory is one of the most celebrated research areas that has an application potential not only in nonlinear but also in several branches of mathematics. As a consequence of this fact, several fixed point results have been reported. It is not easy to know, manage, and use all results of this reich theory to get an application. To overcome such problems and clarify the literature, several authors have suggested a more general construction in a way that a number of existing results turn into a consequence of the proposed one. One of the examples of this trend is the investigations of fixed point of certain operator by using the $\alpha$-admissible mapping introduced Samet et al. [1]. This paper has been appreciated by several authors and this trend has been supported by reporting several interesting results; see for example [2-12].

In this paper, we define $\left(\alpha^{*}, \psi\right)$-contractive multivalued mappings of integral type and discuss the existence of a fixed point of such mappings. Our construction and hence results improve, extend, and generalize several results including Branciari [13] and Feng and Liu [14].

In what follows, we recall some basic definitions, notions, notations, and fundamental results for the sake of completeness. Let $\Psi$ be a family of nondecreasing functions, $\psi:[0, \infty) \rightarrow[0, \infty)$ such that $\sum_{n=1}^{\infty} \psi^{n}(t)<\infty$ for each $t>0$, where $\psi^{n}$ is the $n$th iterate of $\psi$. It is known that, for each $\psi \in \Psi$, we have $\psi(t)<t$ for all $t>0$ and $\psi(0)=0$ for $t=$ 0 [1]. We denote by $\Phi$ the set of all Lebesgue integrable mappings, $\phi:[0, \infty) \rightarrow[0, \infty)$ which is summable on each compact subset of $[0, \infty)$ and $\int_{0}^{\epsilon} \phi(t) d t>0$, for each $\epsilon>0$.

Let $(X, d)$ be a metric space. We denote by $N(X)$ the space of all nonempty subsets of $X$, by $B(X)$ the space of all nonempty bounded subsets of $X$, and by $C L(X)$ the space of all nonempty closed subsets of $X$. For $A \in N(X)$ and $x \in X$,

$$
d(x, A)=\inf \{d(x, a): a \in A\} .
$$

For every $A, B \in B(X)$,

$$
\delta(A, B)=\sup \{d(a, b): a \in A, b \in B\} .
$$

We denote $\delta(A, B)$ by $\delta(x, B)$ when $A=\{x\}$. If, for $x_{0} \in$ $X$, there exists a sequence $\left\{x_{n}\right\}_{n \in \mathbb{N}}$ in $X$ such that $x_{n} \in$ $G x_{n-1}$, then $O\left(G, x_{0}\right)=\left\{x_{0}, x_{1}, x_{2}, \ldots\right\}$ is said to be an orbit of $G: X \rightarrow C L(X)$ at $x_{0}$. A mapping $f:$ $X \rightarrow \mathbb{R}$ is $G$ orbitally lower semicontinuous at $x$, if $\left\{x_{n}\right\}$ is 
a sequence in $O\left(G, x_{0}\right)$ and $x_{n} \rightarrow x$ implies $f(x) \leq$ $\lim \inf _{n} f\left(x_{n}\right)$. Branciari [13] extended the Banach contraction principle [15] in the following way.

Theorem 1. Let $(X, d)$ be a complete metric space and let $G$ : $X \rightarrow X$ be a mapping such that

$$
\int_{0}^{d(T x, T y)} \phi(t) d t \leq c \int_{0}^{d(x, y)} \phi(t) d t
$$

for each $x, y \in X$, where $c \in[0,1)$ and $\phi \in \Phi$. Then $G$ has a unique fixed point.

Since then many authors used integral type contractive conditions to prove fixed point theorems in different settings; see for example [12, 16-22]. Feng and Liu [14] extended the result of Branciari [13] to multivalued mappings as follows.

Theorem 2 (see $[14])$. Let $(X, d)$ be a complete metric space and let $G: X \rightarrow C L(X)$ be a mapping. Assume that for each $x \in X$ and $y \in G x$, there exists $z \in G y$ such that

$$
\int_{0}^{d(y, z)} \phi(t) d t \leq \psi\left(\int_{0}^{d(x, y)} \phi(t) d t\right)
$$

where $\psi \in \Psi$ and $\phi \in \Phi$. Then $G$ has a fixed point in $X$ provided $f(\xi)=d(\xi, G \xi)$ is lower semicontinuous, with $\xi \in X$.

Definition 3 (see [3]). Let $(X, d)$ be a metric space and $\alpha$ : $X \times X \rightarrow[0, \infty)$ be a mapping. A mapping $G: X \rightarrow$ $C L(X)$ is $\alpha^{*}$-admissible if $\alpha(x, y) \geq 1 \Rightarrow \alpha^{*}(G x, G y) \geq 1$, where $\alpha^{*}(G x, G y)=\inf \{\alpha(a, b): a \in G x, b \in G y\}$.

Definition 4 (see [3]). Let $(X, d)$ be a metric space. A mapping $G: X \rightarrow C L(X)$ is called $\alpha^{*}-\psi$-contractive if there exist two functions $\alpha: X \times X \rightarrow[0, \infty)$ and $\psi \in$ $\Psi$ such that

$$
\alpha^{*}(G x, G y) H(G x, G y) \leq \psi(d(x, y))
$$

for all $x, y \in X$.

Theorem 5 (see [3]). Let $(X, d)$ be a complete metric space, let $\alpha: X \times X \rightarrow[0, \infty)$ be a function, let $\psi \in \Psi$ be a strictly increasing map, and let $G$ be a closed-valued $\alpha^{*}$-admissible and $\alpha^{*}-\psi$-contractive multifunction on $X$. Suppose that there exist $x_{0} \in X$ and $x_{1} \in G x_{0}$ such that $\alpha\left(x_{0}, x_{1}\right) \geq 1$. Assume that if $\left\{x_{n}\right\}$ is a sequence in $X$ such that $\alpha\left(x_{n}, x_{n+1}\right) \geq 1$ for all $n$ and $x_{n} \rightarrow x$, then $\alpha\left(x_{n}, x\right) \geq 1$ for all $n$. Then $G$ has a fixed point.

Definition 6 (see [2]). Let $(X, d)$ be a metric space and let $G: X \rightarrow C L(X)$ be a mapping. We say that $G$ is a generalized $\left(\alpha^{*}, \psi\right)$-contractive if there exists $\psi \in \Psi$ such that

$$
\alpha^{*}(G x, G y) d(y, G y) \leq \psi(d(x, y))
$$

for each $x \in X$ and $y \in G x$, where $\alpha^{*}(G x, G y)=\inf \{\alpha(a$, b) : $a \in G x, b \in G y\}$.
Theorem 7 (see [2]). Let $(X, d)$ be a complete metric space and let $G: X \rightarrow B(X)$ be a mapping such that for each $x \in$ $X$ and $y \in G x$, we have

$$
\alpha^{*}(G x, G y) \delta(y, G y) \leq \psi(d(x, y))
$$

where $\psi \in \Psi$. Assume that there exist $x_{0} \in X$ and $x_{1} \in$ $G x_{0}$ such that $\alpha\left(x_{0}, x_{1}\right) \geq 1$. Moreover $G$ is an $\alpha^{*}$-admissible mapping. Then there exists an orbit $\left\{x_{n}\right\}$ of $G$ at $x_{0}$ and $x \in$ $X$ such that $\lim _{n \rightarrow \infty} x_{n}=x$. Moreover, $\{x\}=G x$ if and only if $f(\xi)=\delta(\xi, G \xi)$ is lower semicontinuous at $x$.

\section{Main Results}

In this section, we state and proof our main results. We first give the definition of the following notion.

Definition 8 . Let $(X, d)$ be a metric space. We say that $G$ : $X \rightarrow C L(X)$ is an integral type $\left(\alpha^{*}, \psi\right)$-contractive mapping if there exist two functions $\psi \in \Psi$ and $\phi \in \Phi$ such that for each $x \in X$ and $y \in G x$, there exists $z \in G y$ satisfying

$$
\int_{0}^{\alpha^{*}(G x, G y) d(y, z)} \phi(t) d t \leq \psi\left(\int_{0}^{d(x, y)} \phi(t) d t\right),
$$

where $\alpha^{*}(G x, G y)=\inf \{\alpha(a, b): a \in G x, b \in G y\}$.

Example 9. Let $X=\mathbb{R}$ be endowed with the usual metric $d$. Define $G: X \rightarrow C L(X)$ by

$$
G x= \begin{cases}{[x, \infty)} & \text { if } x \geq 0 \\ (-\infty, 6 x] & \text { if } x<0\end{cases}
$$

and $\alpha: X \times X \rightarrow[0, \infty)$ by

$$
\alpha(x, y)= \begin{cases}x+y+1 & \text { if } x, y \geq 0 \\ 0 & \text { otherwise }\end{cases}
$$

Take $\psi(t)=t / 4$ and $\phi(t)=2 t$ for all $t \geq 0$. Then, for each $x \in X$ and $y \in G x$, there exists $z \in G y$ such that

$$
\int_{0}^{\alpha^{*}(G x, G y) d(y, z)} \phi(t) d t \leq \psi\left(\int_{0}^{d(x, y)} \phi(t) d t\right) .
$$

Hence $G$ is an integral type $\left(\alpha^{*}, \psi\right)$-contractive mapping. Note that (4) does not hold at $x=-2$.

Definition 10. We say that $\phi \in \Phi$ is an integral subadditive if, for each $a, b>0$, we have

$$
\int_{0}^{a+b} \phi(t) d t \leq \int_{0}^{a} \phi(t) d t+\int_{0}^{b} \phi(t) d t .
$$

We denote by $\Phi_{s}$ the class of all integral subadditive functions $\phi \in \Phi$.

Example 11. Let $\phi_{1}(t)=(1 / 2)(t+1)^{-1 / 2}$ for all $t \geq 0, \phi_{2}(t)=$ $(2 / 3)(t+1)^{-1 / 3}$ for all $t \geq 0$, and $\phi_{3}(t)=e^{-t}$ for all $t \geq 0$. Then $\phi_{i} \in \Phi_{s}$, where $i=1,2,3$. 
Definition 12 . Let $(X, d)$ be a metric space. We say that $G$ : $X \rightarrow C L(X)$ is a subintegral type $\left(\alpha^{*}, \psi\right)$-contractive if there exist two functions $\psi \in \Psi$ and $\phi \in \Phi_{s}$ such that for each $x \in X$ and $y \in G x$, there exists $z \in G y$ satisfying

$$
\int_{0}^{\alpha^{*}(G x, G y) d(y, z)} \phi(t) d t \leq \psi\left(\int_{0}^{d(x, y)} \phi(t) d t\right)
$$

where $\alpha^{*}(G x, G y)=\inf \{\alpha(a, b): a \in G x, b \in G y\}$.

Example 13. Let $X=\mathbb{R}$ be endowed with the usual metric $d$. Define $G: X \rightarrow C L(X)$ by

$$
G x= \begin{cases}{\left[\frac{x}{4}, \frac{x}{2}\right]} & \text { if } x \geq 0 \\ {[24 x, 12 x]} & \text { if } x<0\end{cases}
$$

and $\alpha: X \times X \rightarrow[0, \infty)$ by

$$
\alpha(x, y)= \begin{cases}2 & \text { if } x=y=0 \\ 0 & \text { otherwise }\end{cases}
$$

Take $\psi(t)=t / 3$ and $\phi(t)=(2 / 3)(t+1)^{-1 / 3}$ for all $t \geq 0$. Then, for each $x \in X$ and $y \in G x$, there exists $z \in G y$ such that

$$
\int_{0}^{\alpha^{*}(G x, G y) d(y, z)} \phi(t) d t \leq \psi\left(\int_{0}^{d(x, y)} \phi(t) d t\right) .
$$

Hence $G$ is an subintegral type $\left(\alpha^{*}, \psi\right)$-contractive mapping.

Theorem 14. Let $(X, d)$ be a complete metric space and let $G: X \rightarrow C L(X)$ be an $\alpha^{*}$-admissible subintegral type $\left(\alpha^{*}, \psi\right)$-contractive mapping. Assume that there exist $x_{0} \in X$ and $x_{1} \in G x_{0}$ such that $\alpha\left(x_{0}, x_{1}\right) \geq 1$. Then there exists an orbit $\left\{x_{n}\right\}$ of $G$ at $x_{0}$ and $x \in X$ such that $\lim _{n \rightarrow \infty} x_{n}=x$. Moreover, $x$ is a fixed point of $G$ if and only if $f(\xi)=d(\xi, G \xi)$ is $G$ orbitally lower semicontinuous at $x$.

Proof. By the hypothesis, there exist $x_{0} \in X$ and $x_{1} \in$ $G x_{0}$ such that $\alpha\left(x_{0}, x_{1}\right) \geq 1$. Since $G$ is $\alpha^{*}$-admissible, then $\alpha^{*}\left(G x_{0}, G x_{1}\right) \geq 1$. For $x_{0} \in X$ and $x_{1} \in G x_{0}$, there exists $x_{2} \in G x_{1}$ such that

$$
\begin{aligned}
\int_{0}^{d\left(x_{1}, x_{2}\right)} \phi(t) d t & \leq \int_{0}^{\alpha^{*}\left(G x_{0}, G x_{1}\right) d\left(x_{1}, x_{2}\right)} \phi(t) d t \\
& \leq \psi\left(\int_{0}^{d\left(x_{0}, x_{1}\right)} \phi(t) d t\right) .
\end{aligned}
$$

Since $\psi$ is nondecreasing, we have

$$
\psi\left(\int_{0}^{d\left(x_{1}, x_{2}\right)} \phi(t) d t\right) \leq \psi^{2}\left(\int_{0}^{d\left(x_{0}, x_{1}\right)} \phi(t) d t\right)
$$

As $\alpha\left(x_{1}, x_{2}\right) \geq 1$ by $\alpha^{*}$-admissibility of $G$, we have $\alpha^{*}\left(G x_{1}\right.$, $\left.G x_{2}\right) \geq 1$. For $x_{1} \in X$ and $x_{2} \in G x_{1}$, there exists $x_{3} \in$ $G x_{2}$ such that

$$
\begin{aligned}
\int_{0}^{d\left(x_{2}, x_{3}\right)} \phi(t) d t & \leq \int_{0}^{\alpha^{*}\left(G x_{1}, G x_{2}\right) d\left(x_{2}, x_{3}\right)} \phi(t) d t \\
& \leq \psi\left(\int_{0}^{d\left(x_{1}, x_{2}\right)} \phi(t) d t\right) \\
& \leq \psi^{2}\left(\int_{0}^{d\left(x_{0}, x_{1}\right)} \phi(t) d t\right) .
\end{aligned}
$$

Since $\psi$ is nondecreasing, we have

$$
\psi\left(\int_{0}^{d\left(x_{2}, x_{3}\right)} \phi(t) d t\right) \leq \psi^{3}\left(\int_{0}^{d\left(x_{0}, x_{1}\right)} \phi(t) d t\right) .
$$

By continuing the same process, we get a sequence $\left\{x_{n}\right\}$ in $X$ such that $x_{n} \in G x_{n-1}, \alpha\left(x_{n-1}, x_{n}\right) \geq 1$, and

$$
\int_{0}^{d\left(x_{n}, x_{n+1}\right)} \phi(t) d t \leq \psi^{n}\left(\int_{0}^{d\left(x_{0}, x_{1}\right)} \phi(t) d t\right),
$$

for each $n \in \mathbb{N}$.

Letting $n \rightarrow \infty$ in above inequality, we have

$$
\lim _{n \rightarrow \infty} \int_{0}^{d\left(x_{n}, x_{n+1}\right)} \phi(t) d t=0 .
$$

Also, we have

$$
\lim _{n \rightarrow \infty} \int_{0}^{d\left(x_{n}, G x_{n}\right)} \phi(t) d t=0,
$$

which implies that

$$
\lim _{n \rightarrow \infty} d\left(x_{n}, G x_{n}\right)=0 .
$$

For any $n, p \in \mathbb{N}$, we have

$$
d\left(x_{n}, x_{n+p}\right) \leq \sum_{i=n}^{n+p-1} d\left(x_{i}, x_{i+1}\right) .
$$

Since $\phi \in \Phi_{s}$, it can be shown by induction that

$$
\int_{0}^{d\left(x_{n}, x_{n+p}\right)} \phi(t) d t \leq \sum_{i=n}^{n+p-1} \int_{0}^{d\left(x_{i}, x_{i+1}\right)} \phi(t) d t .
$$

From (21) and (26), we have

$$
\int_{0}^{d\left(x_{n}, x_{n+p}\right)} \phi(t) d t \leq \sum_{i=n}^{n+p-1} \psi^{i}\left(\int_{0}^{d\left(x_{0}, x_{1}\right)} \phi(t) d t\right) .
$$

Since $\psi \in \Psi$ it follows that $\left\{x_{n}\right\}$ is Cauchy sequence in $X$. As $X$ is complete, there exists $x^{*} \in X$ such that $x_{n} \rightarrow$ $x^{*}$ as $n \rightarrow \infty$. Suppose $f(\xi)=d(\xi, G \xi)$ is $G$ orbitally lower semicontinuous at $x^{*}$; then

$$
d\left(x^{*}, G x^{*}\right) \leq \liminf _{n} f\left(x_{n}\right)=\lim _{n} \inf d\left(x_{n}, G x_{n}\right)=0 .
$$

By closedness of $G$ it follows that $x^{*} \in G x^{*}$. Conversely, suppose that $x^{*}$ is a fixed point of $G$ then $f\left(x^{*}\right)=0 \leq$ $\lim \inf _{n} f\left(x_{n}\right)$. 
Example 15. Let $X=\mathbb{R}$ be endowed with the usual metric $d$. Define $G: X \rightarrow C L(X)$ by

$$
G x= \begin{cases}{[x, x+1]} & \text { if } x \geq 0, \\ (-\infty, 6 x] & \text { if } x<0,\end{cases}
$$

and $\alpha: X \times X \rightarrow[0, \infty)$ by

$$
\alpha(x, y)= \begin{cases}x+y+1 & \text { if } x, y \geq 0 \\ 0 & \text { otherwise. }\end{cases}
$$

Take $\psi(t)=t / 2$ and $\phi(t)=(1 / 2)(t+1)^{-1 / 2}$ for all $t \geq 0$. Then, for each $x \in X$ and $y \in G x$, there exists $z \in G y$ such that

$$
\int_{0}^{\alpha^{*}(G x, G y) d(y, z)} \phi(t) d t \leq \psi\left(\int_{0}^{d(x, y)} \phi(t) d t\right) .
$$

Hence $G$ is a subintegral type $\left(\alpha^{*}, \psi\right)$-contractive mapping. Clearly, $G$ is $\alpha^{*}$-admissible. Also, we have $x_{0}=1$ and $x_{1}=$ $2 \in G x_{0}$ such that $\alpha\left(x_{0}, x_{1}\right)=4$. Therefore, all the conditions of Theorem 14 are satisfied and $G$ has infinitely many fixed points. Note that Theorem 2 in Section 1 is not applicable here. For example, take $x=-1$ and $y=-6$.

Definition 16. Let $(X, d)$ be a metric space. We say that $G$ : $X \rightarrow B(X)$ is an integral type $\left(\alpha^{*}, \psi, \delta\right)$-contractive mapping if there exist two functions $\psi \in \Psi$ and $\phi \in \Phi$ such that

$$
\int_{0}^{\alpha^{*}(\mathrm{G} x, \mathrm{G} y) \delta(y, \mathrm{G} y)} \phi(t) d t \leq \psi\left(\int_{0}^{d(x, y)} \phi(t) d t\right)
$$

for each $x \in X$ and $y \in G x$, where $\alpha^{*}(G x, G y)=$ $\inf \{\alpha(a, b): a \in G x, b \in G y\}$.

Definition 17. Let $(X, d)$ be a metric space. We say that $G$ : $X \rightarrow B(X)$ is a subintegral type $\left(\alpha^{*}, \psi, \delta\right)$-contractive mapping if there exist two functions $\psi \in \Psi$ and $\phi \in \Phi_{s}$ such that

$$
\int_{0}^{\alpha^{*}(G x, G y) \delta(y, G y)} \phi(t) d t \leq \psi\left(\int_{0}^{d(x, y)} \phi(t) d t\right)
$$

for each $x \in X$ and $y \in G x$, where $\alpha^{*}(G x, G y)=$ $\inf \{\alpha(a, b): a \in G x, b \in G y\}$.

Theorem 18. Let $(X, d)$ be a complete metric space and let $G: \quad X \rightarrow B(X)$ be an $\alpha^{*}$-admissible subintegral type $\left(\alpha^{*}, \psi, \delta\right)$-contractive mapping. Assume that there exist $x_{0} \in X$ and $x_{1} \in G x_{0}$ such that $\alpha\left(x_{0}, x_{1}\right) \geq 1$. Then there exists an orbit $\left\{x_{n}\right\}$ of $G$ at $x_{0}$ and $x \in X$ such that $\lim _{n \rightarrow \infty} x_{n}=x$. Moreover, $x \in X$ such that $\{x\}=G x$ if and only if $f(\xi)=\delta(\xi, G \xi)$ is $G$ orbitally lower semicontinuous at $x$.

Proof. By the hypothesis, there exist $x_{0} \in X$ and $x_{1} \in$ $G x_{0}$ such that $\alpha\left(x_{0}, x_{1}\right) \geq 1$. Since $G$ is $\alpha^{*}$-admissible, then $\alpha^{*}\left(G x_{0}, G x_{1}\right) \geq 1$. For $x_{0} \in X$ and $x_{1} \in G x_{0}$, we have

$$
\int_{0}^{\alpha^{*}\left(G x_{0}, G x_{1}\right) \delta\left(x_{1}, G x_{1}\right)} \phi(t) d t \leq \psi\left(\int_{0}^{d\left(x_{0}, x_{1}\right)} \phi(t) d t\right) .
$$

Since $G x_{1} \neq \emptyset$, then we have $x_{2} \in G x_{1}$ such that

$$
\begin{aligned}
\int_{0}^{d\left(x_{1}, x_{2}\right)} \phi(t) d t & \leq \int_{0}^{\alpha^{*}\left(G x_{0}, G x_{1}\right) \delta\left(x_{1}, G x_{1}\right)} \phi(t) d t \\
& \leq \psi\left(\int_{0}^{d\left(x_{0}, x_{1}\right)} \phi(t) d t\right) .
\end{aligned}
$$

Since $\psi$ is nondecreasing, we have

$$
\psi\left(\int_{0}^{d\left(x_{1}, x_{2}\right)} \phi(t) d t\right) \leq \psi^{2}\left(\int_{0}^{d\left(x_{0}, x_{1}\right)} \phi(t) d t\right) .
$$

As $\alpha\left(x_{1}, x_{2}\right) \geq 1$ by $\alpha^{*}$-admissibility of $G$, we have $\alpha^{*}\left(G x_{1}\right.$, $\left.G x_{2}\right) \geq 1$. Thus, we have $x_{3} \in G x_{2}$ such that

$$
\begin{aligned}
\int_{0}^{d\left(x_{2}, x_{3}\right)} \phi(t) d t & \leq \int_{0}^{\alpha^{*}\left(G x_{1}, G x_{2}\right) \delta\left(x_{2}, G x_{2}\right)} \phi(t) d t \\
& \leq \psi\left(\int_{0}^{d\left(x_{1}, x_{2}\right)} \phi(t) d t\right) \\
& \leq \psi^{2}\left(\int_{0}^{d\left(x_{0}, x_{1}\right)} \phi(t) d t\right) .
\end{aligned}
$$

Since $\psi$ is nondecreasing, we have

$$
\psi\left(\int_{0}^{d\left(x_{2}, x_{3}\right)} \phi(t) d t\right) \leq \psi^{3}\left(\int_{0}^{d\left(x_{0}, x_{1}\right)} \phi(t) d t\right) .
$$

By continuing the same process, we get a sequence $\left\{x_{n}\right\}$ in $X$ such that $x_{n} \in G x_{n-1}, \alpha\left(x_{n-1}, x_{n}\right) \geq 1$, and

$$
\begin{aligned}
\int_{0}^{d\left(x_{n}, x_{n+1}\right)} \phi(t) d t & \leq \int_{0}^{\delta\left(x_{n}, G x_{n}\right)} \phi(t) d t \\
& \leq \psi^{n}\left(\int_{0}^{d\left(x_{0}, x_{1}\right)} \phi(t) d t\right), \\
& \text { for each } n \in \mathbb{N} .
\end{aligned}
$$

Letting $n \rightarrow \infty$ in above inequality, we have

$$
\lim _{n \rightarrow \infty} \int_{0}^{\delta\left(x_{n}, G x_{n}\right)} \phi(t) d t=0,
$$

which implies that

$$
\lim _{n \rightarrow \infty} \delta\left(x_{n}, G x_{n}\right)=0 .
$$

For any $n, p \in \mathbb{N}$, we have

$$
d\left(x_{n}, x_{n+p}\right) \leq \sum_{i=n}^{n+p-1} d\left(x_{i}, x_{i+1}\right) .
$$

Since $\phi \in \Phi_{s}$, it can be shown by induction that

$$
\int_{0}^{d\left(x_{n}, x_{n+p}\right)} \phi(t) d t \leq \sum_{i=n}^{n+p-1} \int_{0}^{d\left(x_{i}, x_{i+1}\right)} \phi(t) d t .
$$


From (39) and (43), we have

$$
\int_{0}^{d\left(x_{n}, x_{n+p}\right)} \phi(t) d t \leq \sum_{i=n}^{n+p-1} \psi^{i}\left(\int_{0}^{d\left(x_{0}, x_{1}\right)} \phi(t) d t\right) .
$$

Since $\psi \in \Psi$ it follows that $\left\{x_{n}\right\}$ is Cauchy sequence in $X$. As $X$ is complete, there exists $x^{*} \in X$ such that $x_{n} \rightarrow$ $x^{*}$ as $n \rightarrow \infty$. Suppose $f(\xi)=\delta(\xi, G \xi)$ is $G$ orbitally lower semicontinuous at $x^{*}$; then

$$
\delta\left(x^{*}, G x^{*}\right) \leq \liminf _{n} f\left(x_{n}\right)=\liminf _{n} \delta\left(x_{n}, G x_{n}\right)=0 .
$$

Hence, $\left\{x^{*}\right\}=G x^{*}$ because $\delta(A, B)=0$ implies $A=B=$ $\{a\}$. Conversely, suppose that $\left\{x^{*}\right\}=G x^{*}$. Then $f\left(x^{*}\right)=0 \leq$ $\lim \inf _{n} f\left(x_{n}\right)$.

Example 19. Let $X=\{1,3,5,7,9, \ldots\}$ be endowed with the usual metric $d$. Define $G: X \rightarrow B(X)$ by

$$
G x= \begin{cases}\{x-2, x+2\} & \text { if } x \neq 1, \\ \{1\} & \text { if } x=1\end{cases}
$$

and $\alpha: X \times X \rightarrow[0, \infty)$ by

$$
\alpha(x, y)= \begin{cases}1 & \text { if } x=y=1 \\ \frac{1}{4} & \text { otherwise }\end{cases}
$$

Take $\psi(t)=t / 2$ and $\phi(t)=(2 / 3)(t+1)^{-1 / 3}$ for all $t \geq$ 0 . Clearly, $G$ is an $\alpha^{*}$-admissible subintegral type $\left(\alpha^{*}, \psi, \delta\right)$ contractive mapping. Also, we have $x_{0}=1$ and $x_{1}=1 \epsilon$ $G x_{0}$ such that $\alpha\left(x_{0}, x_{1}\right)=1$. Therefore, all the conditions of Theorem 18 hold and $G$ has fixed points.

Example 20. Let $X=\mathbb{R}$ be endowed with the usual metric $d$. Define $G: X \rightarrow B(X)$ by

$$
G x= \begin{cases}\{\lfloor x\rfloor,\lceil x\rceil\} & \text { if } x \geq 0, \\ \left(\frac{\lfloor x\rfloor}{4}, \frac{\lceil x\rceil}{2}\right) & \text { if } x<0,\end{cases}
$$

and $\alpha: X \times X \rightarrow[0, \infty)$ by

$$
\alpha(x, y)= \begin{cases}1 & \text { if } x, y \geq 0 \\ 0 & \text { otherwise }\end{cases}
$$

Take $\psi(t)=t / 4$ and $\phi(t)=e^{-t}$ for all $t \geq 0$. Then it is easy to check that all the conditions of Theorem 18 hold. Therefore $G$ has infinitely many fixed points.

Remark 21. Let $\phi(t)=1$ for all $t \geq 0$; Theorem 18 reduces to Theorem 7 in Section 1.

Remark 22. Note that subadditivity of the integral was needed in the proofs of Theorems 14 and 18 in order to obtain inequalities (26) and (43). It is natural to ask wether the conclusions of Theorems 14 and 18 are valid if we replace subintegral contractive conditions (13) and (33) by integral contractive conditions (8) and (32), respectively. Looking at our proofs, we can say that it will be true if the inequalities (26) and (43) hold. Here we would like to mention that many authors (see for example $[14,23]$ ) while proving the results on integral contractions have not assumed that the integral is subadditive but indeed they used the subadditivity of the integral in the proofs of their results while obtaining the inequalities comparable to inequalities (26) and (43).

\section{Application}

In this section, we obtain some fixed point results for partially ordered metric spaces, as consequences of aforementioned results. Moreover, we apply our result to prove the existence of solution for an integral equation.

Let $A$ and $B$ be subsets of a partially ordered set. We say that $A \preceq_{r} B$, if for each $a \in A$ and $b \in B$, we have $a \preceq b$.

Theorem 23. Let $(X, \preceq, d)$ be a complete ordered metric space and let $G: X \rightarrow C L(X)$ be a mapping such that for each $x \in$ $X$ and $y \in G x$ with $x \preceq y$, there exists $z \in G y$ satisfying

$$
\int_{0}^{d(y, z)} \phi(t) d t \leq \psi\left(\int_{0}^{d(x, y)} \phi(t) d t\right),
$$

where $\psi \in \Psi$ and $\phi \in \Phi_{s}$. Assume that there exist $x_{0} \in X$ and $x_{1} \in G x_{0}$ such that $x_{0} \preceq x_{1}$. Also, assume that $x \preceq$ $y$ implies $G x \preceq_{r} G y$. Then there exists an orbit $\left\{x_{n}\right\}$ of $G$ at $x_{0}$ and $x \in X$ such that $\lim _{n \rightarrow \infty} x_{n}=x$. Moreover, $x$ is a fixed point of $G$ if and only if $f(\xi)=d(\xi, G \xi)$ is $G$ orbitally lower semicontinuous at $x$.

Proof. Define $\alpha: X \times X \rightarrow[0, \infty)$ by

$$
\alpha(x, y)= \begin{cases}1 & \text { if } x \preceq y \\ 0 & \text { otherwise }\end{cases}
$$

By using hypothesis of corollary and definition of $\alpha$, we have $\alpha\left(x_{0}, x_{1}\right)=1$. As $x \preceq y$ implies $G x \preceq_{r}$ Gy, by using the definitions of $\alpha$ and $\preceq_{r}$, we have that $\alpha(x, y)=1$ implies $\alpha^{*}(G x, G y)=1$. Moreover, it is easy to check that $G$ is an integral type $\left(\alpha^{*}, \psi\right)$-contractive mapping. Therefore, by Theorem 14, there exists an orbit $\left\{x_{n}\right\}$ of $G$ at $x_{0}$ and $x \in X$ such that $\lim _{n \rightarrow \infty} x_{n}=x$. Moreover, $x$ is a fixed point of $G$ if and only if $f(\xi)=d(\xi, G \xi)$ is $G$ orbitally lower semicontinuous at $x$.

Considering $G: X \rightarrow X$ and $\phi(t)=1$ for each $t \geq 0$, Theorem 23 reduces to following result.

Corollary 24. Let $(X, \preceq, d)$ be a complete ordered metric space and let $G: X \rightarrow X$ be a nondecreasing mapping such that, for each $x \in X$ with $x \preceq G x$, we have

$$
d\left(G x, G^{2} x\right) \leq \psi(d(x, G x))
$$

where $\psi \in \Psi$. Assume that there exists $x_{0} \in X$ such that $x_{0} \preceq$ $G x_{0}$. Then there exists an orbit $\left\{x_{n}\right\}$ of $G$ at $x_{0}$ and $x \in$ $X$ such that $\lim _{n \rightarrow \infty} x_{n}=x$. Moreover, $x$ is a fixed point of $G$ if and only if $f(\xi)=d(\xi, G \xi)$ is $G$ orbitally lower semicontinuous at $x$. 
Consider an integral equation of the form

$$
x(t)=\int_{a}^{b} K(t, s, x(s)) d s, \quad t \in[a, b],
$$

where $K:[a, b] \times[a, b] \times \mathbb{R} \rightarrow \mathbb{R}$ is continuous and nondecreasing.

\section{Theorem 25. Assume that}

(i) for $u, v \in C([a, b], \mathbb{R})$, with $u(t) \leq v(t)$ for each $t \in$ $[a, b]$, we have

$$
|K(t, s, u(t))-K(t, s, v(t))| \leq \frac{\psi(d(u, v))}{(b-a)}
$$

for each $t, s \in[a, b]$, where $\psi \in \Psi$;

(ii) for each $t, s \in[a, b]$, there exists $x_{0} \in C([a, b], \mathbb{R})$ such that

$$
x_{0}(t) \leq \int_{a}^{b} K\left(t, s, x_{0}(s)\right) d s
$$

Then there exists an iterative sequence $\left\{x_{n}\right\}$, starting from $x_{0}$, and $x \in C([a, b], \mathbb{R})$ such that $\lim _{n \rightarrow \infty} x_{n}=x$. Moreover, $x$ is a solution of (53) if and only if $f(\xi)=d(\xi, y)$ is lower semicontinuous at $x$, where $y(t)=\int_{a}^{b} K(t, s, \xi(s)) d s$.

Proof. It is easy to see that $X=C([a, b], \mathbb{R})$ is complete with respect to the metric $d(x, y)=\max _{t \in[a, b]}|x(t)-y(t)|$. We define partial ordering on $X$ as follows: $x \preceq y$ if and only if $x(t) \leq y(t)$ for each $t \in[a, b]$. Define $G: X \rightarrow$ $X$ by $G x=y$, where $y(t)=\int_{a}^{b} K(t, s, x(s)) d s$, for each $t, s \in$ $[a, b]$. From (ii), we have $x_{0} \preceq G x_{0}$. For $x \in X$, let $G x=$ $y$ and $G y=z$; that is, $y(t)=\int_{a}^{b} K(t, s, x(s)) d s$ and $z(t)=$ $\int_{a}^{b} K(t, s, y(s)) d s$, for each $t, s \in[a, b]$. Then, for each $x \in$ $X$ with $x \preceq G x$, we have

$$
\begin{aligned}
d\left(G x, G^{2} x\right) & =\max _{t \in[a, b]}|y(t)-z(t)| \\
& =\max _{t \in[a, b]} \mid \int_{a}^{b} K(t, s, x(s)) d s \\
& \quad-\int_{a}^{b} K(t, s, y(s)) d s \mid \\
\leq & \max _{t \in[a, b]} \int_{a}^{b}|K(t, s, x(s))-K(t, s, y(s))| d s \\
\leq & \frac{\psi(d(x, G x))}{(b-a)}(b-a) .
\end{aligned}
$$

That is $d\left(G x, G^{2} x\right) \leq \psi(d(x, G x))$, for each $x \in X$ with $x \preceq$ $G x$. Clearly, $G$ is nondecreasing. Therefore, all conditions of Corollary 24 hold and the conclusions follow from Corollary 24.

\section{Conflict of Interests}

The authors declare that there is no conflict of interests regarding the publication of this paper.

\section{Authors' Contribution}

All authors contributed equally and significantly in writing this paper. All authors read and approved the final paper.

\section{Acknowledgment}

The authors are grateful to the reviewers for their careful readings and useful comments.

\section{References}

[1] B. Samet, C. Vetro, and P. Vetro, "Fixed point theorems for $\alpha$ $\psi$-contractive type mappings," Nonlinear Analysis, vol. 75, no. 4, pp. 2154-2165, 2012.

[2] M. U. Ali and T. Kamran, "On $\left(\alpha^{*}, \psi\right)$-contractive multivalued mappings," Fixed Point Theory and Applications, vol. 2013, article 137, 2013.

[3] J. H. Asl, S. Rezapour, and N. Shahzad, "On fixed points of $\alpha-\psi$-contractive multifunctions," Fixed Point Theory and Applications, vol. 2012, article 212, 2012.

[4] G. Minak and I. Altun, "Some new generalizations of Mizoguchi-Takahashi type fixed point theorem," Journal of Inequalities and Applications, vol. 2013, article 493, 2013.

[5] E. Karapinar, H. Aydi, and B. Samet, "Fixed points for generalized $(\alpha, \psi)$-contractions on generalized metric spaces," Journal of Inequalities and Applications, vol. 2014, article 229, 2014.

[6] M. U. Ali, T. Kamran, and E. Karapinar, "A new approach to $(\alpha, \psi)$-contractive nonself multivalued mappings," Journal of Inequalities and Applications, vol. 2014, p. 71, 2014.

[7] M. U. Ali, Q. Kiran, and N. Shahzad, "Fixed point theorems for multi-valued mappings involving $\alpha$-function," Abstract and Applied Analysis, vol. 2014, Article ID 409467, 6 pages, 2014.

[8] M. U. Ali, T. Kamran, and N. Shahzad, "Best proximity point for $\alpha-\psi$-proximal contractive multimaps," Abstract and Applied Analysis. In press.

[9] M. U. Ali, T. Kamran, and E. Karapinar, "Fixed point of $\alpha$ $\psi$-contractive type mappings in uniform spaces," Fixed Point Theory and Applications. Accepted.

[10] E. Karapinar, "Discussion on $\alpha-\psi$ contractions on generalized metric spaces," Abstract and Applied Analysis, vol. 2014, Article ID 962784, 7 pages, 2014.

[11] E. Karapinar and B. Samet, "Generalized $\alpha-\psi$ contractive type mappings and related fixed point theorems with applications," Abstract and Applied Analysis, vol. 2012, Article ID 793486, 17 pages, 2012.

[12] E. Karapinar, P. Shahi, and K. Tas, "Generalized $\alpha$ - $\psi$-contractive type mappings of integral type and related fixed point theorems," J Inequal. Appl, vol. 2014, article 160, 2014.

[13] A. Branciari, "A fixed point theorem for mappings satisfying a general contractive condition of integral type," International Journal of Mathematics and Mathematical Sciences, vol. 29, no. 9, pp. 531-536, 2002.

[14] Y. Feng and S. Liu, "Fixed point theorems for multi-valued contractive mappings and multi-valued Caristi type mappings," 
Journal of Mathematical Analysis and Applications, vol. 317, no. 1, pp. 103-112, 2006.

[15] S. Banach, "Sur les operations dans les ensembles abstraits et leur application aux equations integrales," Fundamenta Mathematicae, vol. 3, pp. 133-181, 1922.

[16] V. Popa, "A general fixed point theorem for occasionally weakly compatible mappings and applications," Scientific Studies and Research: Series Mathematics and Informatics, vol. 22, no. 1, pp. 77-92, 2012.

[17] B. Samet and C. Vetro, "An integral version of Ćirić's fixed point theorem," Mediterranean Journal of Mathematics, vol. 9, no. 1, pp. 225-238, 2012.

[18] T. Kamran, "Fixed point theorems for hybrid mappings satisfying an integral type contractive condition," Georgian Mathematical Journal, vol. 19, no. 1, pp. 117-125, 2012.

[19] W. Sintunavarat and P. Kumam, "Gregus-type common fixed point theorems for tangential multivalued mappings of integral type in metric spaces," International Journal of Mathematics and Mathematical Sciences, vol. 2011, Article ID 923458, 12 pages, 2011.

[20] I. Altun and D. Turkoglu, "Some fixed point theorems for weakly compatible multivalued mappings satisfying some general contractive conditions of integral type," Bulletin of the Iranian Mathematical Society, vol. 36, no. 1, pp. 55-67, 2010.

[21] H. K. Pathak and N. Shahzad, "Gregus type fixed point results for tangential mappings satisfying contractive conditions of integral type," Bulletin of the Belgian Mathematical Society, vol. 16, no. 2, pp. 277-288, 2009.

[22] S. Chauhan, W. Shatanawi, S. Radenovic, and I. Abu-Irwaq, "Variants of sub-sequentially continuous mappings and integral-type fixed point results," Rendiconti del Circolo Matematico di Palermo, vol. 63, no. 1, pp. 53-72, 2014.

[23] P. Vijayaraju, B. E. Rhoades, and R. Mohanraj, "A fixed point theorem for a pair of maps satisfying a general contractive condition of integral type," International Journal of Mathematics and Mathematical Sciences, no. 15, pp. 2359-2364, 2005. 


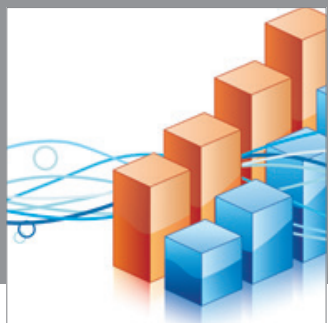

Advances in

Operations Research

mansans

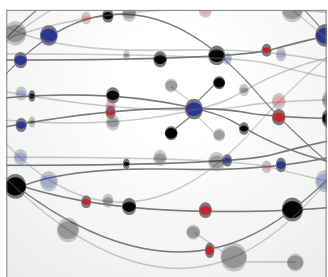

The Scientific World Journal
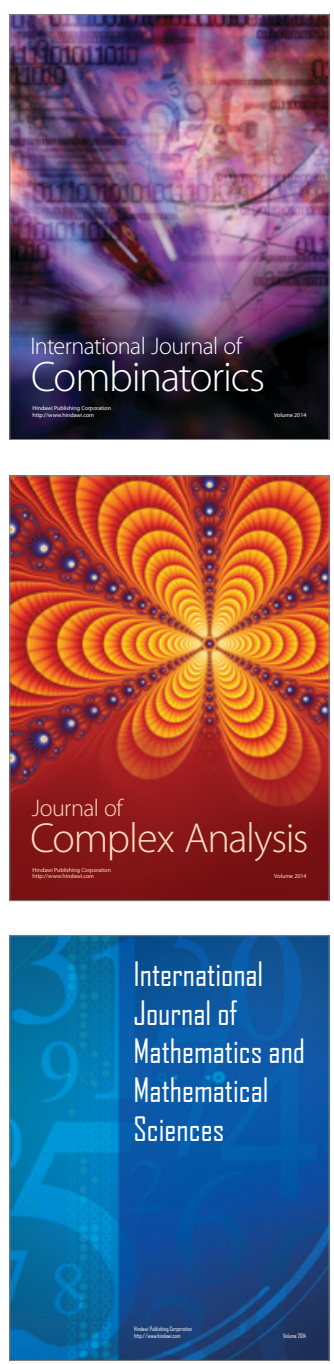
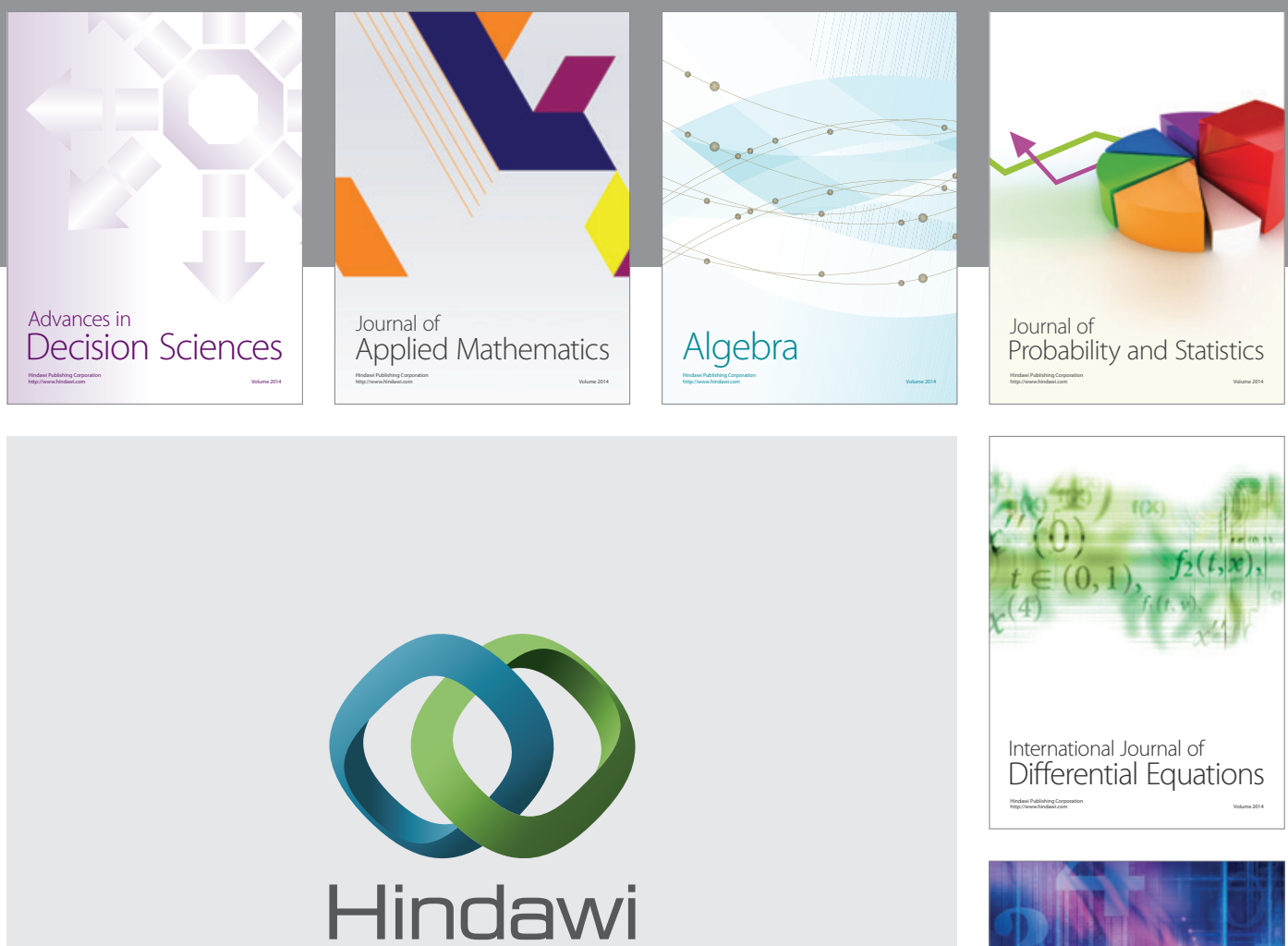

Submit your manuscripts at http://www.hindawi.com
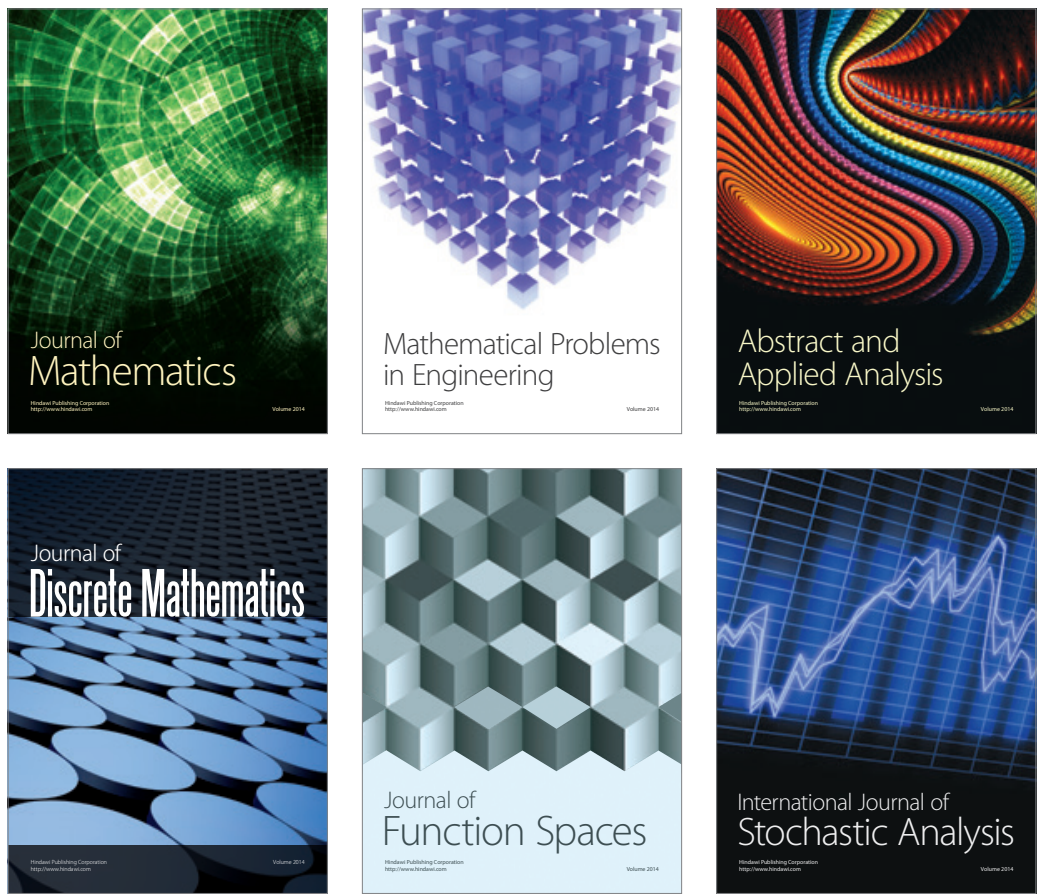

Journal of

Function Spaces

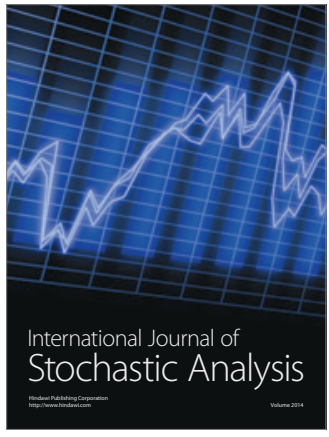

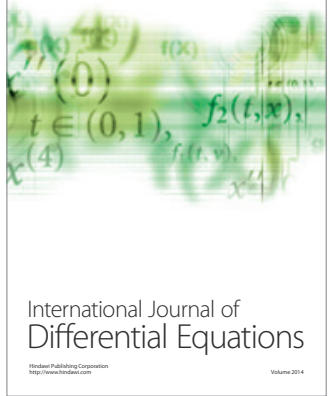
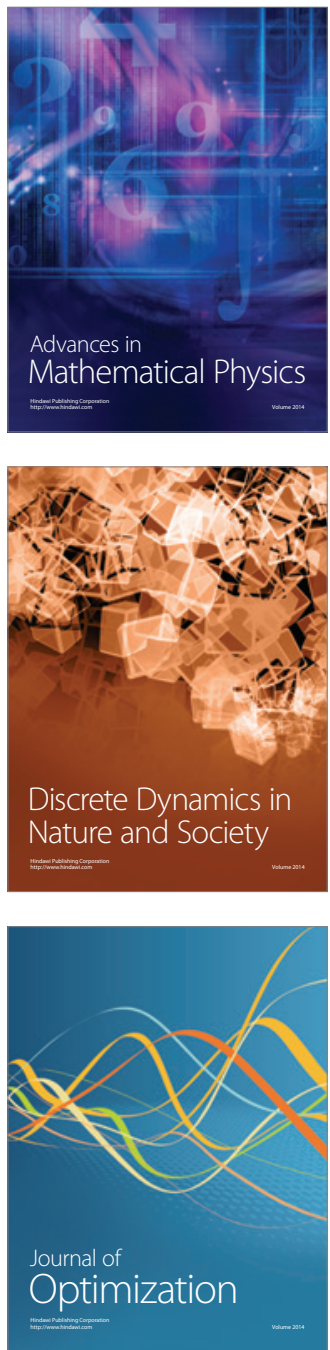